\title{
Magnetic Regulator Modeling
}

\author{
Jeff Vollin, F. Dong Tan, S. M. Cuk \\ Power Electronics Group, 116-81, Caltech \\ Pasadena, CA 91125 \\ Tel: (818) 356-4835, Fax: (818) 356-2944
}

\begin{abstract}
A novel method of providing regulation in a resonant power supply uses a device called a magnetic regulator which resembles an ordinary $A C$ transformer. Through the use of additional control windings, the conversion ratio of the magnetic regulator can be electronically varied thus achieving control. A circuit model for the magnetic regulator is derived and is shown to contain a current-controlled leakage inductance. Measurements are made on an experimental device which verify the model. Two additional structures are described including a new invention which has significant advantages over the prior art.
\end{abstract}

\section{INTRODUCTION}

In the area of high voltage power supply (HVPS) design, many significant improvements have been made in recent years with respect to weight and size by utilizing high frequency switching techniques. In areas where weight and size are at a premium such as aircraft or missile radar, the switching frequencies have been driven above $300 \mathrm{kHz}$ in designs utilizing square wave switching techniques. Often, the requirement to operate at such high switching frequencies comes not from a consideration of the power supply's weight or volume, but from a system requirement to synchronize the switching frequency of the power supply to some system clock, to reduce the harmful effects caused by the switching frequency and its many harmonics. In these supplies, the switching losses in the semiconductors and particularly the high voltage diodes become excessive. The designer then finds that the efficiency (and therefore the internal device temperatures) must be sacrificed to operate at these high frequencies. One way to alleviate some of the problems associated with these high switching frequencies is to use one of the many resonant switching techniques.

While resonant switching techniques allow higher frequency power supplies to operate efficiently, conventional designs have several disadvantages. One of

This work was sponsored by Hughes Missile Systems Group, Canoga Park, CA

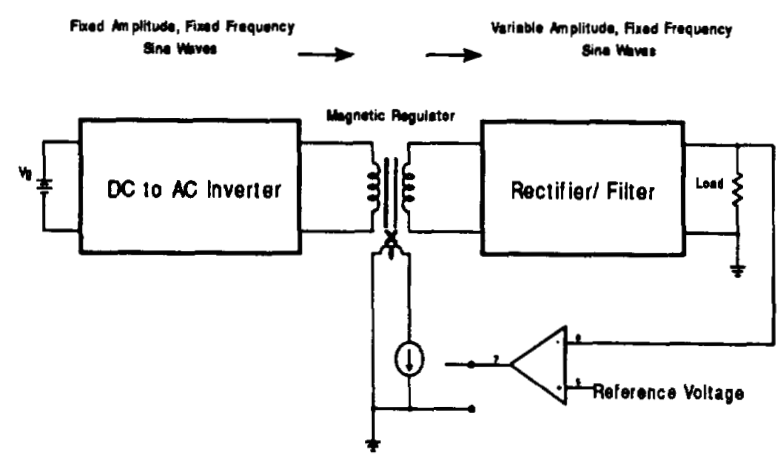

Fig. 1. Block Diagram of a DC-to-DC Power Converter with Fixed Frequency regulation using a Magnetic Regulator.

the major disadvantages is the fact that regulation of the output voltage is accomplished by varying the switching frequency. Obviously, a variable switching frequency cannot be synchronized to a fixed system clock, so some other mechanism is needed to accomplish the regulation.

Recently, a novel device called a magnetic regulator (MR) was proposed in [1], in which control over a sinusoidal output voltage, at a fixed frequency, is possible in response to a controlling current. This device appears at first glance to be a transformer with an electronicallyadjustable turns ratio. In fact, as shown below, the MR will function as a transformer if the control current that drives it is fixed. Further, if one varies the control current, the input to output conversion ratio is modified. Since the device is ideally lossless (real devices suffer from conductor loss in the windings and core loss in the core), the MR can function as a kind of 'control valve' for sinusoidal power signals. This suggests that a power supply with high efficiency could be constructed using an MR in a way analogous to the pass element in a linear power supply.

By embedding this device into a resonant DC-to-DC converter such as described in [2], regulation of the output 
voltage is still possible but now at a fixed switching frequency. A simple block diagram is shown in Fig. 1 to illustrate the idea.

While the converter described above works well, there is no theory available to describe its operation. This paper will take the MR as a separate device and develop an equivalent circuit model based upon the actual physical structure of the magnetic device. In the model, each of the components can be directly related back to physical properties of the device. This results in a circuit model which provides both an intuitive feel for the device's operation and a quantitative model suitable for calculations. Once the modeling method is established, another structure is introduced and its shortcomings identified. This leads naturally to a new structure with significant advantages.

\section{PHYSICAL OPERATION}

The physical structure of one type of magnetic regulator is illustrated in Fig. 2. The core is similar to an EI core except with four legs instead of three. The legs are numbered one through four from left to right. A winding we shall call the primary or input winding is wound around leg number three and another winding, the secondary, is wound around leg number four. These windings can have different numbers of turns. Legs 1 and 2 each have windings wound around them with equal numbers of turns and then seriesconnected in such a way that the flux produced by the primary will cancel in the series combination of these windings.. A DC current source is connected to the series combination of coils 1 and 2 and thereby controls the DC flux density in the left half of the structure. For this reason, these windings are referred to as the control windings. Leg 4 has a small intentional air-gap.

If an AC source is connected to the primary terminals, then the flux generated by the primary winding will flow in leg 3 and then divide between the left and right paths according to the reluctance associated with each path. If there is no DC current in the control windings, the path to the left will have much lower reluctance than the path to the right owing to the air-gap placed in leg 4 . In this case, very little flux will flow into leg 4 and therefore very little $A C$ voltage will be induced in the secondary windings. Further, there will be no $\mathrm{AC}$ voltage developed at control winding terminals because of the series-opposing connection of the windings.

Now if a DC current large enough to saturate legs 1 and 2 is driven into the control windings, the reluctance of the path to the left of the primary increases greatly, forcing the AC flux from the primary to 'steer' to the secondary leg through the air-gap. In this case, the voltage induced on the secondary will be equal to the primary voltage times the turns ratio of the secondary to the primary, less some lost

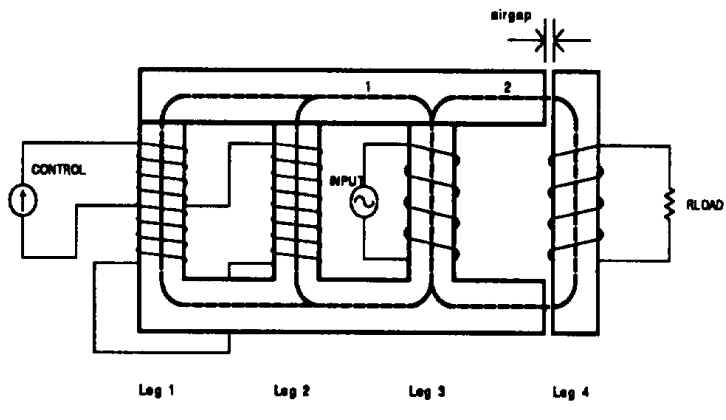

Fig. 2. Physical Structure of the Four-Leg Magnetic Regulator.

voltage due to the inevitable leakage of some of the primary flux into the air surrounding the core.

The above describes the extremes of the control range. Between zero and maximum control current, the permeability is varied continuously so that the conversion ratio is varied continuously. In addition, the core material is usually soft ferrite which provides a smooth variation in the conversion ratio. Regulation can then be easily obtained using linear feedback concepts. The next section will quantify the concepts developed above to calculate the flux distribution in the core and therefore the input-output behavior of the device.

\section{MODELING}

\section{The Reluctance Model}

Since a knowledge of the flux distribution in the core of the MR is necessary to calculate the terminal voltages, the first problem to attack is that of the magnetic circuit. Interestingly, a method used on a very different problem in power electronics is directly applicable here. In [3], the problem of the design of an integrated coupled inductor was solved by using the reluctance concept to generate a magnetic equivalent circuit which then could be used to design an appropriate inductor. The $\mathrm{AC}$ waveforms in [3] were square waves and the operation of the power processing system was quite different, however the reluctance model used there applies equally well to this case.

To model a magnetic device using the reluctance concept, one first identifies the important magnetic paths which make up the device. Each of these paths is assigned a 'magnetic resistance' or, precisely, a reluctance. Each of the windings of the device will appear as magnetic 'voltage sources' whose voltage is equal to the ampere-tums product. The unknown quantity is the magnetic 'current' which is the 
flux in the actual device. The flux is solved for using standard electric circuit analysis techniques. An equivalent circuit for the magnetic device which will model its terminal characteristics can be directly obtained from the magnetic equivalent circuit. This is accomplished by recognizing that the inductance is inversely proportional to the reluctance and that terminal currents are proportional to the voltage sources in the magnetic equivalent circuit. Similarly, the 'currents' (flux) in the magnetic equivalent circuit are proportional to the voltage in the electric circuit. This implies that the electric circuit model can be derived from the magnetic circuit model using duality, where the reluctances are replaced by inductances. To properly account for the constants involved, each winding in the actual device appears in the electric equivalent circuit as an ideal transformer. A more complete description of this method is described in [4].

Fig. 3 illustrates the various reluctance elements that make up the MR superimposed on the core geometry to emphasize the relationship between the elements in the reluctance model and the actual physical structure. The diagram is based upon the experimental four-leg MR which was assembled from six pieces of ferrite material. While this model is specific to the experimental device, it will be

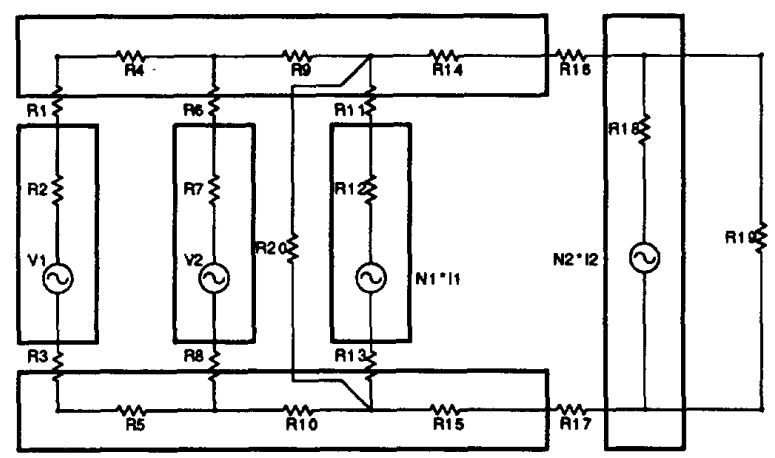

Fig. 3. Reluctance Model for the Four-Leg Magnetic Regulator.

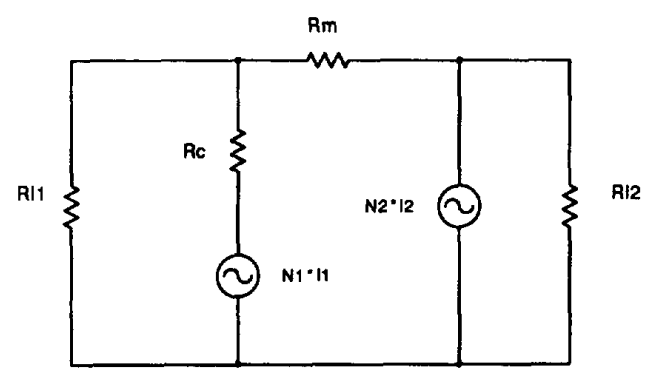

Fig. 4. Simplified reluctance model simplified in Fig. 4 to a more general model which is common to all the MR's designed to date. The primary and secondary windings are illustrated as magnetomotive force sources $\mathrm{N}_{1} \mathrm{I}_{1}$ and $\mathrm{N}_{2} \mathrm{I}_{2}$ respectively.

Several of the reluctances in this case are physically identical which will simplify the calculations considerably. Reluctances R1, R3, R6, R8, R11, and R13 represent the residual air-gap that inevitably results when separate pieces of ferrite are used. This gap is as small as possible, and is estimated to be $2.54 \mu \mathrm{m}$ (.0001 in). Reluctances R2, R7, and $R 12$ are all the same being made from identical pieces of core material. Similarly, R4, R5, R9, R10, R14, and R15 are identical ferrite paths. $R 16$ and $R 17$ represent the intentional air-gap introduced into the structure for control purposes. Each gap is $190 \mu \mathrm{m}$ (.0075 in). Reluctance R18 is unique due to the geometry used for leg 4 . This reluctance is neglected as small in the final model. R19 and R20 are the leakage fluxes in air and therefore quite large (but measurable.)

Fig. 3 can be simplified to Fig. 4 by straightforward combinations of the elements in Fig. 3 . Reluctance $R_{l 1}$ is the sum total of all of the flux which does not pass through the intentional air-gaps. This is the total primary leakage flux. $R_{l 2}$ is the secondary leakage flux path and is equal to $R 19$. The coupling flux path is modeled by $R_{m}$. Reluctance $R_{c}$ is the combination of all the reluctances which carry the common flux of both the leakage and coupling paths. The values are:

$$
\begin{aligned}
& R_{t 1}=R 20\left(\left(R 9+R 10+\left(\frac{(R 1+R 2+R 3+R 4+R 5)+(R 6+R 7+R 8)}{2}\right)\right)\right. \\
& R_{c}=R 11+R 12+R 13 \\
& R_{m}=R 14+R 15+R 16+R 17 \\
& R_{L 2}=R 19
\end{aligned}
$$

The reluctance $R_{c}$ is usually quite small and would normally be neglected when compared to other elements in the model. It will be shown in the measurements section however that this element will be required to properly account for the measured behavior. Note that there is no DC source shown for the control winding mmf. The effect of the control windings is to modulate the permeability of the control legs so the reluctances R2, R4, R5 and R7 are functions of the control current from the general definition of the reluctance:

$$
R=\frac{l}{\mu S}
$$

Where $R$ is the reluctance of a bar of length $l$, cross-sectional area $S$, and permeability $\mu$. 
Since the permeability is a function of the control current, so is the reluctance. Note that the flux paths through each of the control legs have substantially different reluctances owing to the greater distance of leg 1 from the primary (leg 3 ) than leg 2 . This would cause an AC voltage to appear across the series-opposing connection of the control windings. This is undesirable since the control current source is usually a transistor collector which should not be reverse biased. To prevent the AC voltage from appearing across the control winding terminals, a capacitor is used which effectively shorts the AC voltage. This places the control windings in parallel thus forcing equal $\mathrm{AC}$ voltage on each. This implies that the flux through legs 1 and 2 are forced to be equal at a cost of some circulating current in the control windings. The voltage sources V1 and $\mathrm{V} 2$ in Fig. 3 are therefore equal in magnitude and opposite in polarity. $R_{l 1}$ is computed with this in mind in (1). In computing $R_{l 1}$ the dependent sources V1 and V2 are accounted for, so no AC ampere-turns are needed to represent the control windings in the simplified model.

\section{The Inductance Model}

The next step in modeling the MR is to transform the equivalent magnetic circuit into a circuit model for the inductances. By using the duality of the electric and

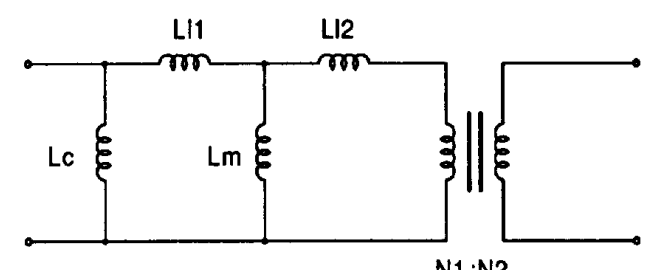

$N 1: N 2$

Fig. 5. Inductance Model for the Four-Leg Magnetic Regulator.

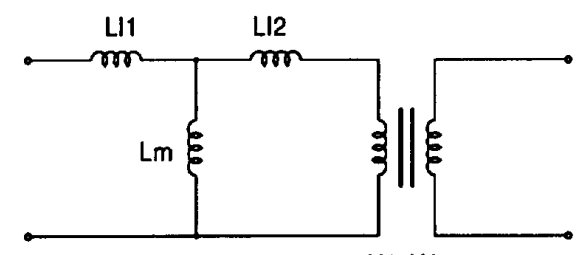

N1:N2

Fig. 6. Minimum-Inductance Equivalent Circuit for a Two-winding device. magnetic fields as described above, the equivalent electric circuit in Fig. 4 is transformed to that shown in Fig. 5. The same subscripts are used in each Fig. with all the R's replaced by L's. The L's are calculated as follows:

$$
L_{i}=\frac{N^{2}}{R_{i}}
$$

Where $\mathrm{N}$ is chosen to be the number of turns on the primary winding ( this choice is arbitrary; in general each mmf source is replaced by a pair of terminals and an ideal transformer whose turns ratio is $\mathrm{N}_{\mathrm{a}}: \mathrm{N}_{b}$ where $\mathrm{N}_{\mathrm{a}}$ is a reference number of turns and $\mathrm{N}_{b}$ is the number of turns of the mmf source winding. Using $\mathrm{N}_{\mathrm{a}}=\mathrm{N}_{\mathrm{b}}$ inserts a $1: 1$ transformer at the primary terminals which is then neglected. This choice of $\mathrm{N}_{\mathrm{a}}$ amounts to referencing all the inductances in the model to the primary.)

The model shown in Fig. 5 is very similar to the usual model of a two-winding transformer with the exception of the element $L_{c}$. As stated above, $L_{c}$ is actually present in a conventional transformer, but is usually neglected. The most significant prediction from the model is that the turns ratio of the ideal transformer is fixed as are the inductances except for $L_{l 1}$. $L_{l 1}$ is proportional to the permeability of the control legs and hence would decrease with increasing control current. Measurements done below show this behavior clearly.

\section{EXPERIMENTAL VERIFICATION}

An experimental magnetic regulator was constructed using the dimensions shown in Table 1 . The measurements were done using a computer-controlled network analyzer to measure the $\mathrm{AC}$ quantities and a computer-controlled power supply to sweep the control current during the measurement. The individual inductances must be extracted from the data measured at the terminals of the device. Since the magnetic regulator is being treated as a two port device, there are 4 parameters which can be determined from terminal measurements. Further, the magnetic regulator is a reciprocal device so only 3 of the parameters are independent. Since the model for the magnetic regulator has 4 elements, they cannot be uniquely determined by terminal measurements alone. This means only a 3-inductor equivalent circuit can be uniquely specified by measurements and therefore the equivalent circuit of Fig. 6 can be used as a basis. The measurements are of three types: 1) Input Inductance with the secondary open $\left(L_{11, \text { open }}\right)$. 2) Output inductance with the primary open $\left(L_{22, \text { open }}\right)$. 3) Secondary / primary ratio, driving the primary with the secondary open $\left(R_{21, \text { open }}\right)$. The model quantities in Fig. 6 can be obtained from the measured quantities as in (4). 
TABLE 1. DIMENSIONS OF THE FOUR-LEG MAGNETIC REGULATOR.

\begin{tabular}{|l|lll|}
\hline Element & Length $(\mathrm{cm})$ & Area $(\mathrm{cm} 2)$ & $\begin{array}{l}\text { Reluctance } \\
(1 / \mathrm{H})\end{array}$ \\
\hline $\begin{array}{l}\text { Top, } \\
\text { Bottom Bar } \\
\text { Legs 1,2,3 }\end{array}$ & 75.9 & 0.87 & $116 \mathrm{k}$ \\
Leg 4 & 27.4 & 1.18 & $92.4 \mathrm{k}$ \\
$\begin{array}{l}\text { Residual } \\
\text { air-gap } \\
\begin{array}{l}\text { Intentional } \\
\text { air-gap }\end{array}\end{array}$ & 0.00025 & 1.18 & $17.1 \mathrm{k}$ \\
\hline
\end{tabular}

$$
\begin{aligned}
& L_{l 1}=R_{21, \text { open }} L_{11, \text { open }} \\
& L_{m}=L_{11 \text { open }}-L_{l 1} \\
& L_{l 2}=L_{22, \text { open }}-L_{l 1}
\end{aligned}
$$

The ideal transformer's turns ratio is obtained from the winding information and is assumed to be 1:1 for simplicity in the present context.

Fig. 7 shows the measurements for the case where $L_{c}$ is ignored as is described by the above equations. From Fig. 7 it is apparent that the inductances $L_{m}$ and $L_{l 2}$ are not in fact independent of control current as was postulated above. The reason for this behavior is that the inductance $L_{c}$ was ignored. Even though this inductor cannot be directly measured from terminal measurements, its presence is very real and it disturbs the measurements of the other inductances. To account for $L_{c}$, the approach taken was to calculate $L_{c}$ from the geometry of the center leg, and use that value as an input into the measurement routine. If the value for $L_{c}$ is correct, and the proposed model is correct, then the inductances $L_{m}$ and $L_{12}$ should become independent of control current at the same time. The equations for the magnetic regulator model parameters with $L_{c}$ included now become:

$$
\begin{aligned}
& L_{x}=\frac{L_{c} L_{11, \text { open }}}{L_{c}-L_{11, \text { open }}} \\
& L_{m}=R_{21, \text { open }} L_{x} \\
& L_{n 1}=L_{x}-L_{m} \\
& L_{l 2}=L_{22, \text { open }}-L_{m} \|\left(L_{n}+L_{c}\right)
\end{aligned}
$$
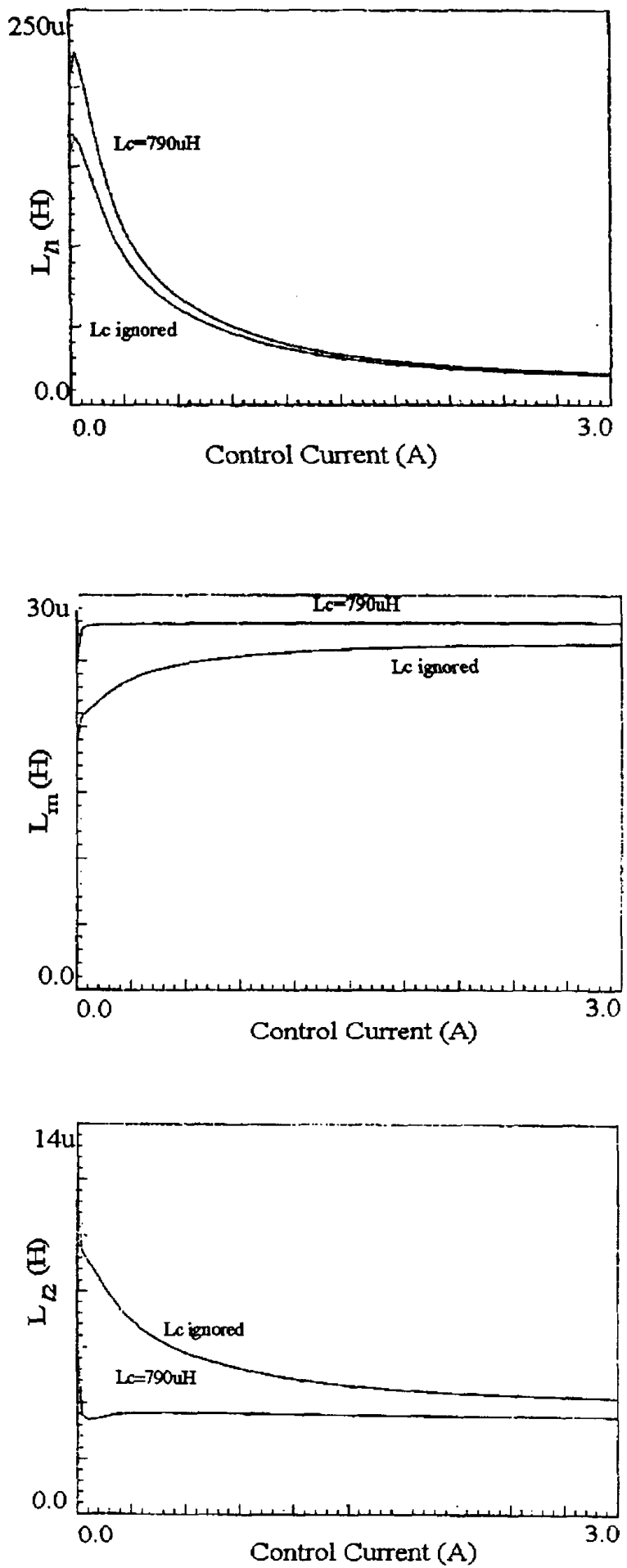

Fig. 7. Inductances Extracted from Measurements showing the effect of including inductor Lc. 
Using the dimensions of the center leg from Table 1 the reluctance of the center leg is $92.4 \mathrm{k}$ plus the air-gap reluctance of $34.2 \mathrm{k}$ yield a total value for $R_{c}$ to be $126.6 \mathrm{k}$. The corresponding inductance is $790 \mu \mathrm{H}$. Using this value for $L_{c}$, the other inductances can be recalculated as shown in fig 7. As predicted, the same value that gave a flat curve for $L_{m}$ also gave a flat curve for $L_{l}$. The fact that a single value for $L_{c}$ makes both $L_{m}$ and $L_{R}$ independent of control current is proof that the model is correct.

The reluctance concept also predicts values for the other elements in the model. The value for $L_{l 1}$ takes on a range of values from the value for the zero-bias ferrite permeability of approximately 2000 down to a value determined by the leakage fluxes in the air. The air leakage flux can be estimated from the measured value for $\mathrm{L}_{l 2}$. This value of inductance yields a reluctance value for a winding on a single leg as suggested in Fig. 3, R19. This reluctance value, $27.9 \times 10^{6} 1 / \mathrm{H}$, can then be divided by three to obtain a rough value for $\mathrm{R} 20$ which is $9.3 \times 10^{6} 1 / \mathrm{H}$. This translates to an inductance of $10.8 \mu \mathrm{H}$. This assumes that the saturated reluctance of $R 2$ and $R 7$ go to infinity which is of course not realistic. Nevertheless, the predicted value could be taken as a lower bound on the estimate for $L_{l 1}$ at high control currents. The predicted values for the model elements are summarized in table 2

\section{OTHER MAGNETIC REGULATOR STRUCTURES}

The magnetic regulator discussed above is one of several possible structures for this device. In [1], another possible structure is described which uses two E-I cores, side-by-side. The control windings are wound around one of the outer legs of each core separately, then the two cores are placed side-by-side and the primary wound around the center legs of both cores. Similarly, the secondary is wound around the unused outer legs of both cores. Fig. 8 shows two views of the device along with the path of the control flux.

In this structure, the control legs are equidistant from the primary so there is no circulating current in the control winding as was present in the four-leg magnetic regulator described above. The reluctance model for this device is very similar to the previous case with one less leg. The magnetic circuit is shown in Fig. 9. Note the absence of the voltage sources in the control leg as compared to Fig. 3. This is due to the fact that the flux divides equally between the two control legs naturally, without any circulating current in the control windings. The complete reluctance model in Fig. 9 can be reduced to the simplified model shown in Fig. 4 by straight-forward circuit manipulations. In this case, however, $R_{l 1}$ is not the only reluctance that is a function of control current; this time $R_{c}$ is also dependent upon the control current since the DC control flux travels through it. The practical implication of this is that it is no
TABLE 2. PREDICTED VERSUS MEASURED VALUES FOR THE MAGNETIC REGULATOR

\begin{tabular}{|c|cc|}
\hline Element & Predicted $(\mu \mathrm{H})$ & Measured $(\mu \mathrm{H})$ \\
\hline$L_{l 1}$ & $283-10.8$ & $225-20$ \\
$\mathrm{~L}_{m}$ & 26.8 & 28 \\
$\mathrm{~L}_{l 2}$ & - & 3.58 \\
$\mathrm{~L}_{c}$ & 790 & $\begin{array}{c}\text { Provides flat } L_{m} \\
\text { and } L_{l 2}\end{array}$ \\
\hline
\end{tabular}

longer possible to specify a single value for $\mathrm{L}_{\mathrm{c}}$ and then extract the other inductances with a measurement. Also, as shown in [5], the core loss in ferrite which contains a DC bias is greater than that in unbiased ferrite. This means the core loss in the primary leg would be greater than that in the four-leg structure.

These two shortcomings were the motivation for a new structure described in detail in [6]. In this device, the core is a custom-made core which resembles an E-I core with a longitudinal slot cut in one of the outer legs. A sketch of the device is shown in Fig. 10. The windings are wound as in the case of the three-leg magnetic regulator. The absence of a central gap confines the DC control flux to just the control legs of the core and this eliminates the variation of $R_{c}$, and therefore $L_{c}$, so the inductance values can easily be
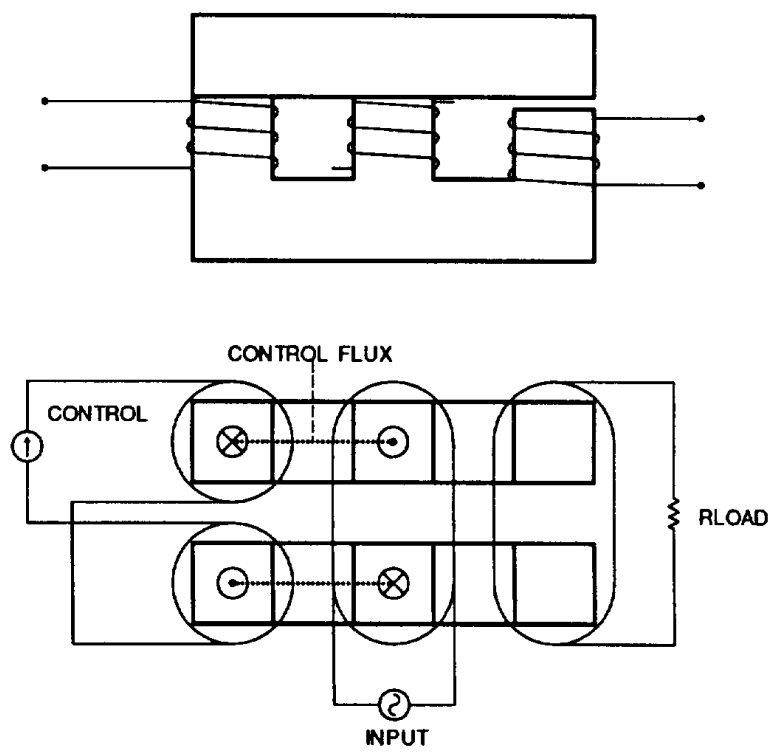

Fig. 8. Top view of the three-leg magnetic regulator, showing the core and winding configuration and the control flux path. 


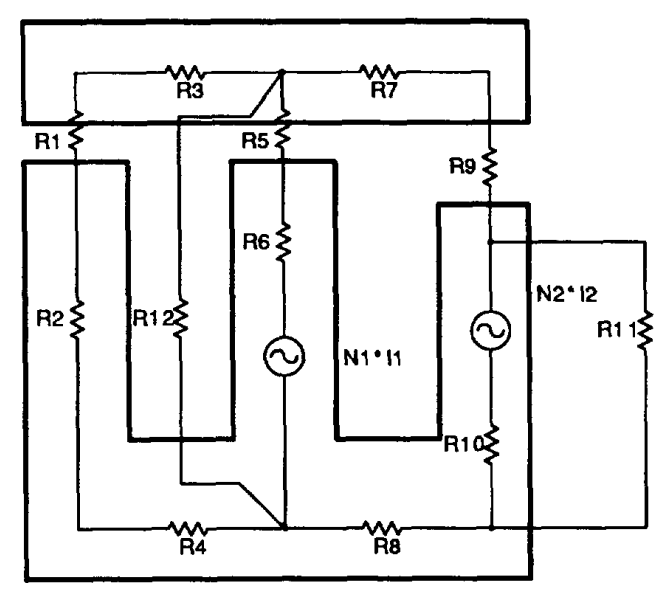

Fig. 9. Reluctance model for the three-leg magnetic regulator.

extracted from measurements as in the four-leg case. In addition, the DC flux occupies much less of the total volume of the core as compared to the three-leg magnetic regulator, yielding lower core losses. This new device therefore combines the best features of each of the devices from [1] without the undesirable features. A photograph of an experimental model of the improved magnetic regulator is shown in Fig. 11.

\section{CONCLUSION}

In this paper a magnetic regulating device has been characterized which is capable of operation within a resonant converter to regulate the output voltage at a fixed frequency. The device resembles a transformer with an electronically controlled leakage inductance. An equivalent circuit for the device is developed which both qualitatively and quantitatively describes the performance of the device. Measurements made on one particular design of the magnetic regulator verify the validity of the model.

The simplified reluctance model for the magnetic regulator presented in Fig. 4 contains all the important elements needed to describe the behavior of the magnetic regulator and also maintains a relationship between the equivalent circuit model and the physical structure of the device. This model is simple enough to be included in a larger analysis of the operation of a power processing system using the magnetic regulator as a regulating element. Such an analysis could be used to design an appropriate compensation network for the feedback loop in the system.

The first magnetic regulator analyzed in this paper has one disadvantage in that a circulating current in the control windings exists in order that the $\mathrm{AC}$ voltage across the winding is zero. A second structure was discussed which
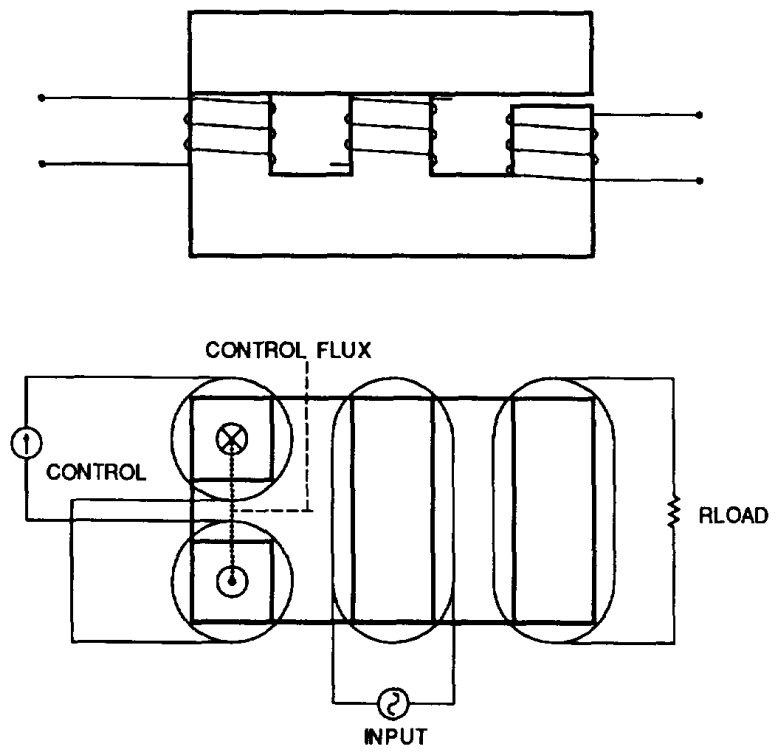

Fig. 10. The basic structure of an improved 3-leg magnetic regulator

eliminates the circulating current but at a cost of increased core loss and an equivalent circuit with two variable elements rather than one. The problems with these structures then motivated the invention of a new structure which captures the desirable features of the previous devices without the disadvantages.

\section{ACKNOWLEDGMENTS}

The authors would like to thank $\mathrm{R}$. Rosen and $\mathrm{K}$. Friedenthal at Hughes Aircraft Company, Missile Systems Group for sponsorship of this research. 


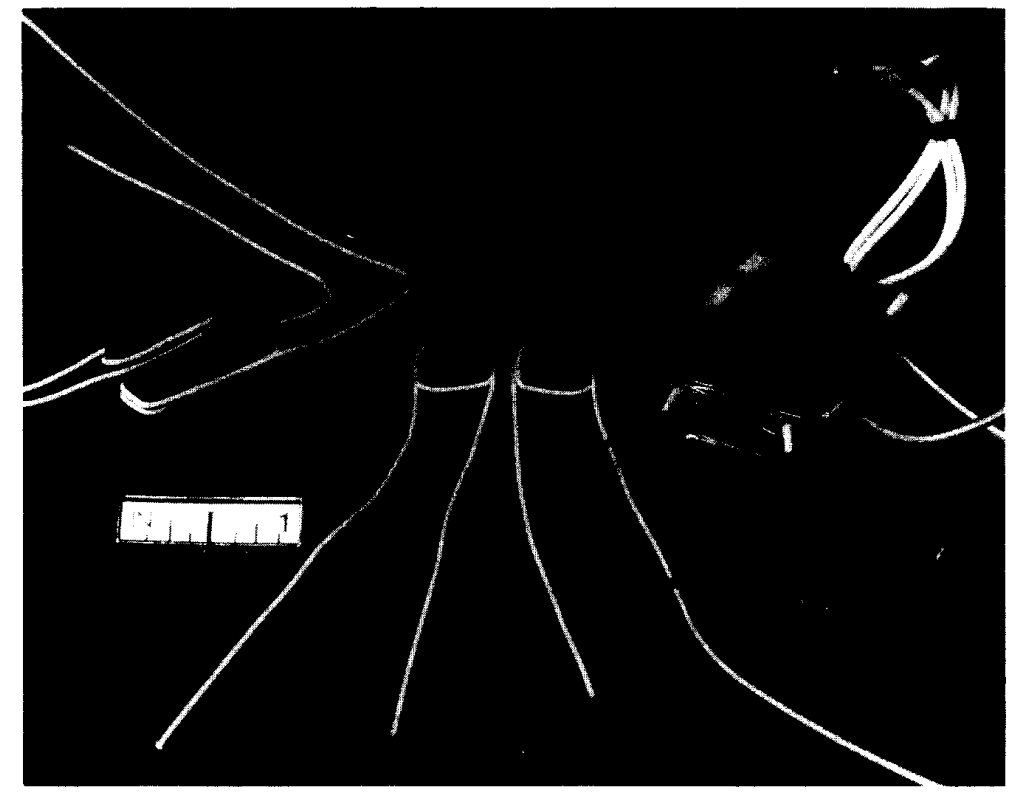

Fig. 11. An experimental model of the improved magnetic regulator. The completed device is on the right. One half of the core containing the primary and secondary windings is on the left. The other half of the core containing the control windings is in the center. The windings on the latter two core halves are for illustration purposes only. This model was designed for a 10V, $1 \mathrm{~A}$ power supply with $10 \mathrm{kV}$ primary-to-secondary isolation.

\section{REFERENCES}

[1] R. D. Washburn and R. F. McClanahan, "Non-Saturating Magnetic Amplifier Controller," U.S. Patent, \#4,841,428 June 20, 1989.

[2] R. Redl, B. Molnar, and N. Sokal, "Class E resonant regulated DC/DC power converters: analysis of operations, and experimental results at 1.5 MHz," IEEE Transactions on Power Electronics, Vol PE-1, No. 2, April, 1986

[3] S. Cuk and Z. Zhang, "Coupled-inductor analysis and design," IEEE PESC Record, 1986, pp. 655-665.

[4] J. K. Watson, "Applications of Magnetism," John Wiley, New York, 1980

[5] F. Dong Tan, J. Vollin, and S. Cuk, "A practical approach for magnetic core loss characterization," IEEE APEC Record, 1993.

[6] J. Vollin, F. Dong Tan, "Improvement to Non-Saturating Magnetic Amplifier Controller" U. S. Patent Application filed Dec, 1992. 